Revista

Actualidades Investigativas

en Educación

\title{
Formación en lectura de cuentos: Impacto en intervenciones en educadoras docentes y no docentes
}

Story Reading Training: Impact on interventions of teacher and nonteacher educators

\section{Volumen 19, Número 3 \\ Setiembre-Diciembre \\ pp. 1-32}

María Soledad Manrique

Citar este documento según modelo APA

Manrique, María Soledad. (2019). Formación en lectura de cuentos: Impacto en intervenciones en educadoras docentes y no docentes. Revista Actualidades Investigativas en Educación, 19(3), 1-32. Doi. 10.15517/aie.v19i3.38631 


\section{Formación en lectura de cuentos: Impacto en intervenciones en educadoras docentes y no docentes \\ Story Reading Training: Impact on interventions of teacher and non-teacher educators}

\section{María Soledad Manrique ${ }^{1}$}

Resumen: Con el fin de estudiar los cambios que la formación promueve, la investigación compara el impacto de una instancia formativa de cuatro meses de duración, en las intervenciones durante la lectura de cuentos de dos grupos de educadoras de infantes en situación de pobreza en Argentina: un grupo de educadoras no docentes, otro de educadoras docentes. Las educadoras no docentes son cinco madres cuidadoras, con un nivel educativo bajo y una formación específica de carácter informal. Las educadoras docentes son 5 maestras tituladas con varios años de ejercicio de su rol. Ambos grupos de mujeres atienden una población infantil de 3 a 5 años de sectores vulnerables. El dispositivo de formación diseñado cumplió con un doble objetivo de investigación y de formación. Permitió, por un lado, estudiar los procesos de cambio en las participantes. Por otro lado, tuvo como fin promover en las participantes el desarrollo de habilidades que dieran lugar a un andamiaje durante la lectura de cuentos que potenciara el desarrollo lingüístico y cognitivo infantil. Contó con dos instancias: una grupal y una individual. La instancia grupal se desarrolló mediante la metodología de taller con una serie de actividades tales como: dramatizaciones de situaciones de lectura, visualizaciones en video de maestras leyendo cuentos, exposiciones en las que se presentaron nuevos conceptos acerca de los procesos cognitivos infantiles para comprender un cuento y sus dificultades y ejercicios que permitían ensayar posibles modos de intervención para facilitar dichos procesos. En la instancia individual se realizó una filmación de cada docente leyendo un cuento y se transcribió la situación filmada y se analizó esa situación junto con la docente. El corpus se armó con las 10 situaciones de lectura de cuentos anteriores (5 de cada grupo de educadoras) y las 10 posteriores al dispositivo de formación. Se empleó el método comparativo constante en la elaboración de un sistema de categorías para clasificar las predicaciones del habla extra-textual de ambos grupos de educadoras durante la lectura de cuentos, de acuerdo a la demanda de procesamiento cognitivo planteado en cada predicación. La información obtenida permitió codificar las intervenciones de las educadoras. Los resultados del análisis cuantitativo en que se pondera la frecuencia de aparición de las categorías analizadas en cada situación, muestran diferencias importantes entre ambos grupos durante las situaciones de lectura previas al curso. Muestran también cómo estas diferencias en la intervención entre ambos grupos de educadoras se reducen luego de la formación. Se complementaron estos resultados con la descripción de los cuentos y el de la dinámica de lectura. Concluimos que, en tanto el aumento de las intervenciones con alta demanda cognitiva para infantes -observado en ambos grupos-, es mayor para las educadoras no docentes, el dispositivo parece haber tenido mayor impacto en ese grupo, que en el de docentes. Discutimos las implicancias de este hallazgo en términos de la lectura de un cuento versus narración oral, y también en relación con el diseño del dispositivo de formación, su capacidad transformadora y las condiciones de posibilidad de formarse de todas las personas.

Palabras clave: educación permanente, formación docente preescolar, lectura de cuentos, grupos desfavorecidos

\footnotetext{
1 Profesora de la Universidad de Buenos Aires, Argentina. Investigadora adjunta de CONICET. ORCID: https://orcid.org/00000001-7206-5629
}

Dirección electrónica: solemanrique@yahoo.com.ar

Artículo recibido: 14 de marzo, 2019

Enviado a corrección: 23 de mayo, 2019

Aprobado: 15 de julio, 2019 


\begin{abstract}
In order to study the changes that the training promotes, the research compares the impact of a formative four months term project, in the interventions during the reading of stories by two educator groups of infants living in poverty in Argentina : one group of non-teaching educators, other of teacher educators. The non-teaching educators are five caregivers, with a low level of education and specific training of an informal nature. The teacher educators are 5 qualified teachers with several years exercising their role. Both groups of caregiver women for a child population from 3 to 5 years old from vulnerable sectors. The designed training device fulfilled a double objective of research and training. On the one hand, it allowed, to study the processes of change in the participants. On the other hand, it aimed to promote in the participants the development of skills which would rise a platform during the reading of stories that will enhance the linguistic and cognitive development of children. It had two samples: a group and an individual. The sample groups were developed through the workshop methodology with a series of activities such as follow: Dramatizations of reading situations, video visualisations of teachers reading stories, exhibitions in which new concepts were presented about children cognitive processes to understand a story and its difficulties and exercises that allowed to test possible ways of intervention to facilitate these processes. In the individual sample, a film was performed by each teacher reading a story and the filmed situation was transcribed then such situation was analyzed together with the teacher. The corpus was set up with the 10 situations of reading of previous stories (5 of each group of educators) and the 10 subsequent to the training device. The constant comparative method was used in the elaboration of a system by categories to classify the predications of the extra-textual speech of both groups of educators during the reading of stories, according to the demand of cognitive processing raised in each preaching. The obtained information allowed to codify the educators interventions. The results of the quantitative analysis in which the frequency of appearance of the analyzed categories is balanced in each situation, show important differences between both groups during the reading situations prior to the course. They also show how these differences in the intervention between both groups of educators are reduced after the training. These results were complemented by the description of the stories and the reading dynamics. We conclude that, while the increase in interventions with high cognitive demand for infants-observed in both groups is greater for non-teaching educators, the device seems to have had greater impact in this group than in the one of the teachers. We discuss the implications of this finding in terms of reading a story versus oral narration, and also in relation to the design of the training device, its transformative capacity and the conditions of possibility for training in all the persons.
\end{abstract}

Key Words: lifelong education, preschool teacher education, story reading, disadvantaged groups

\title{
1. Introducción
}

Como parte de un proyecto de investigación sobre la formación docente continua en Argentina ${ }^{2}$, que tiene por objeto de estudio las transformaciones subjetivas y el cambio en el desempeño a partir de la formación, nos proponemos comparar el desempeño durante la lectura de cuentos compartida en salas de Jardín de Infantes de dos grupos de educadoras docentes y no docentes-, antes y después de su participación en una instancia de formación específicamente diseñada para favorecer el desarrollo infantil en la Provincia de Buenos Aires.

La relevancia de la comparación entre estos dos grupos de educadoras radica en dos cuestiones, una empírica y otra teórica. Desde el punto de vista empírico, es una realidad en Argentina que la educación de la niñez de sectores urbano-marginados se encuentra, en muchos casos, a cargo de educadoras no docentes a las que también se conoce con el nombre de "madres cuidadoras" (De aquí en adelante: MC).

\footnotetext{
2 "Formación docente y transformación subjetiva. El cambio en los modelos mentales y en la práctica pedagógica en el Nivel Inicial." CONICET - CIIPME (2011 - 2019)
} 
La figura de MC existe en toda América Latina, denominada también madre comunitaria o mamá del corazón. Surgida desde los años 80 en un contexto de deterioro social y de déficit de oferta estatal, las MC son reclutadas de entre las familias en situación de pobreza para hacerse cargo de grupos de infantes de su propia comunidad, a cambio de un salario. Inicialmente se trató de una tarea que realizaban en sus propios hogares, y poco a poco fueron incorporándose a sistemas educativos no formales. Con mayor o menor apoyo y capacitación otorgada por diferentes organismos gubernamentales y no gubernamentales, programas comunitarios o de desarrollo social, las MC actualmente se desempeñan en numerosos países como: Guatemala, Perú, Uruguay, Panamá, México, Colombia, Ecuador, Bolivia, Paraguay, Argentina, entre otros.

En Argentina las MC son seleccionadas dentro de los barrios carenciados en los que están situadas las escuelas, por su calidad humana y por su predisposición. Su nivel socioeconómico y educativo es muy bajo: pocas han concluído sus estudios secundarios. La formación específica para el desempeño de este rol es informal y depende en gran medida de la institución en la que se desempeñan, de instancias informales o de sus iniciativas autodidactas, pues al carecer de título secundario se ven excluidas de cualquier instancia de formación oficial.

Ahora bien, para la niñez de sectores sociocultural y económicamente desfavorecidos, el pasaje por el Nivel Inicial significa la oportunidad de acceder a una serie de códigos comunicativos propios de la escuela y de la cultura letrada, de desarrollar una serie de habilidades que contribuyen con su permanencia en el sistema educativo y que permiten achicar la brecha con infantes de sectores de nivel sociocultural mas alto (Siraj-Blatchford y Manni, 2008; Siraj-Blatchford, 2010). En este sentido, las MC cumplen con una función pedagógica fundamental para el desarrollo de este grupo de infantes. Por todo esto, es necesario conocer los contextos de aprendizaje que ofrecen este grupo de educadoras que consideramos desatendido en comparación con el de docentes con titulación e indagar el efecto que tiene la formación en ellas.

Desde el punto de vista teórico, la relevancia de la comparación del desempeño en una actividad tan relevante como la lectura de cuentos (Piacente y Tittarelli, 2009) entre estos dos grupos de educadoras de diferentes niveles socioeconómicos y culturales y el cambio a partir de la formación, puede significar una contribución a la producción de conocimiento en dos campos. En primer lugar, en torno a las particularidades que la formación como proceso de transformación conlleva para diferentes grupos de sujetos en formación. En segundo 
lugar, en relación con la lectura de cuentos en sí misma, como actividad promotora del desarrollo infantil.

Presentamos, a continuación, el marco teórico del trabajo, una breve descripción del dispositivo, la metodología y los resultados del análisis de las situaciones de lectura de cuentos antes y después del dispositivo de formación. Elaboramos algunas hipótesis acerca de los cambios que tuvieron lugar en las docentes. Concluimos el trabajo con una discusión acerca de las implicancias de los resultados.

\section{Marco Teórico y Antecedentes}

\subsection{La formación de educadoras}

En el proyecto del cual este trabajo forma parte hemos ido elaborando una definición multirreferenciada (Souto, 2016) de la formación. Esto significa que abordamos la formación desde diferentes perspectivas teóricas que iluminan aspectos distintos de este objeto complejo consideramos tanto los procesos intra como los intersubjetivos que conlleva.

Como proceso intrasubjetivo, desde una perspectiva psíquica, entendemos que la formación supone transformación del self y del falso self docente (Winnicot, 1986). Esta transformación es posible cuando tiene lugar un retorno sobre sí que permite una elaboración psíquica. Esto es posible a través de lo que denominamos un proceso de análisis (Blanchard- Laville, 2013).

Si enfocamos este proceso intrasubjetivo desde una perspectiva cognitiva, atenderemos a la modificación de esquemas de percepción, valoración y acción que conforman los modelos mentales (Mevorach y Strauss, 2012) de las docentes. El retorno sobre sí como trabajo de la formación es definido en el caso de estas líneas como reflexión (Perrenoud, 2004).

Tanto desde las perspectivas psicoanalíticas como de las cognitivas, se entiende que este proceso intrasubjetivo se apoya en un proceso intersubjetivo: la mediación de un tercero.

Más alla de lo dicho, partimos de la hipótesis de que cualquiera que sea la dimensión que elijamos enfocar en este proceso, la transformación subjetiva que le es propia, atravesada en situaciones reales por demandas institucionales y sociales, se traduce -de modo no lineal - en el caso de educadoras, en nuevas prácticas pedagógicas, que pueden significar mayores oportunidades de aprendizaje infantil (Girolametto, Weitzman y Greenberg, 2003; Neuman y Cunningham, 2009). 
Ahora bien, siguiendo a Pozo et al. (2006) entendemos que las prácticas pedagógicas se basan en conocimiento implícito - no consciente para quien lo emplea-, altamente automatizado y naturalizado, que guía la toma de decisiones sin ser cuestionado. Este conocimiento adquirido a través de la experiencia y de la detección de regularidades cumple una función pragmática que consiste en simplificar el ambiente para poder dar respuestas rápidas y eficaces. No está ligado a la comprensión, sino a la capacidad de predecir y de controlar el ambiente. Incluso para algunos autores (Pozo et al., 2006), este conocimiento se organiza en forma de teorías implícitas que pueden convivir con otras explicitas y conscientes, aun cuando sean contradictorias. Por todo esto es muy difícil de modificar. Quienes estudian las dificultades de la formación suponen que este puede ser uno de los motivos que explican que no siempre se logre la transformación esperada y que exista tan poca relación entre los títulos y cursos docentes y la educación de calidad brindada a la niñez (Assen, Meijers, Otting y Poell 2016).

A partir de lo antes dicho acerca de la necesidad de una formación permanente o contínua, nuestro marco teórico se apoya en trabajos que han mostrado que hablar acerca de las propias acciones y del desempeño real en el aula es más productivo que discutir con las docentes acerca de sus creencias sobre la enseñanza (Hattie, 2009). Es por eso que estos estudios sugieren la observación y la reflexión sistemática sobre el propio desempeño como métodos apropiados para la formación (Brouwer y Korthagen, 2005; DarlingHammond, 2006). Nuestro trabajo comparte esta línea y emplea video-filmaciones, al igual que otros trabajos (Barth-Cohen, Little y Abrahamson, 2018; Gelfuso y Dennis, 2017). En nuestro caso, se registra el propio desempeño en el aula, sobre el cual cada educadora realiza un auto -análisis (Manrique y Sanchez Troussel, 2017) que le permite, entre otras cosas, inferir las creencias que subyacen y que sostienen su desempeño.

Teniendo en cuenta las teorías mencionadas hipotetizamos, previo al estudio, que las maestras (M) tendrían mayor facilidad que las madres cuidadoras (MC) para reflexionar sobre sus prácticas y, por tanto, su desempeño se transformaría más que el de las MC. Supusimos que el haber atravesado por un trayecto de formación en pedagogía habría implicado un mayor desarrollo de su capacidad reflexiva y de la toma de conciencia del propio modo de actuar. Suponíamos, complementariamente, que el grado de automatismo de las teorías implícitas sería mayor para las MC por no haber tenido la oportunidad de revisar sus propios fundamentos durante su formación. 
Nos formulamos las siguientes preguntas que serán respondidads en el trabajo: ¿Qué características toma la intervención docente durante la lectura de cuentos antes del dispositivo de formación en dos grupos de educadoras docentes (M) y no docentes (MC) a cargo de infantes en situación de pobreza? ¿En qué aspectos se modifican estas intervenciones luego de la participación en un dispositivo de formación diseñado específicamente para favorecer el desarrollo lingüístico y cognitivo infantil? ¿Cuáles son las diferencias entre ambos grupos antes y después del dispositivo de formación? ¿Qué nos pueden indicar estas diferencias en relación con la formación de ambos grupos de educadoras? ¿Qué nos puede estar indicando esta información en relación con la situación de lectura de cuentos como actividad promotora del desarrollo infantil? En este trabajo, ofrecemos las respuestas que hemos elaborado a estas preguntas, a partir del análisis.

\subsection{Los intercambios maestra - infantes en situaciones de lectura de cuentos}

Existe acuerdo en la literatura con respecto a la relevancia que tiene el habla adulta para el desarrollo lingüístico y cognitivo infantil (Dickinson y Porche, 2011; Justice, Mashburn, Hamre y Pianta, 2008), particularmente durante la lectura de cuentos (Borzone, 2005; Justice, Weber, Ezell y Bakeman, 2002; Piacente y Tittarelli, 2009; Hammett Price, van Kleeck y Huberty, 2009; Raikes et al., 2006). Los estudios especializados en el tema han elaborado hipótesis diferentes para explicar qué aspecto particular de la lectura compartida de cuentos es el que potencia el desarrollo. En este punto interesa detenernos. Es precisamente en el modo de leer y en los intercambios en torno a la lectura en donde la formación puede afectar a quienes se encuentran a cargo del aprendizaje de infantes y promover, así, mejores oportunidades de desarrollo para ellos y ellas.

Los estudios que constituyen antecedentes de nuestra investigación han concluido que el efecto potenciador del desarrollo sería el diálogo extendido que tiene lugar entre persona adulta e infantes (Wasik, Bond y Hindman, 2006; Lonigan y Whitehurst, 1998; Whitehurst et al.,1994), el cual puede tomar la forma de un andamiaje de alta calidad (Justice et al., 2008; Mashburn, Justice, Downer y Pianta, 2009; Pence, Justice y Wiggins, 2008; Pentimonti y Justice, 2010; Van Kleeck, Vander Woude y Hammett, 2006). Algunos estudios han indicado que este andamiaje de calidad se revela en el hecho de que maestra e infantes puedan involucrarse en una conversación con sentido (Bateman, 2013).

Algunos trabajos describen las intervenciones adultas que parecen impactar en el desarrollo infantil como todas aquellas en las que se emplea lenguaje descontextualizado y 
conceptos abstractos (Dickinson y Smith, 1994; Curenton y Justice, 2004; Justice, Meier y Walpole, 2005; Rosemberg y Silva, 2009; van Kleeck et al., 2006; Wasik et al., 2006). Otros han ido más alla; explican que lo que favorece el desarrollo consiste en la posibilidad que brinda la situación de lectura de ir más allá del aquí y ahora, y que permite que el estudiantado realice inferencias, razone, hipotetice, imagine, prediga y resuelva problemas (Kintsch, 2005).

Otros estudios enfocan el tema del desarrollo infantil identificado explícitamente las intervenciones o estrategias adultas que pueden optimizarlo (Massey, Pence, Justice y Bowles, 2008; Rosemberg y Silva, 2009; Wasik et al., 2006). Dentro del grupo de trabajos que estudian las intervenciones adultas, algunos se han enfocado en lo que llaman habla extendida o habla extra-textual durante la lectura de cuentos. Han advertido la particular relevancia que tienen las preguntas formuladas por docentes, notando que las que son abiertas estimulan la realización de inferencias, y por tanto, la comprensión y el "pensamiento compartido sostenido" (Siraj-Blatchford y Manni, 2008). Devescovi y Baumgartner (1993) agregan que el intento de responder a cada pregunta genera una actividad mental que reestructura y reorganiza las representaciones mentales. Massey et al. (2008), elaboraron una tipología de preguntas enn función de la demanda cognitiva en 14 clases de preescolar de sectores desfavorecidos. Estudiaron si variaba la frecuencia de estos tipos de preguntas en las aulas de acuerdo a las actividades realizadas. Las preguntas de menor demanda cognitiva son las que se focalizan en aspectos observables y las de mayor demanda cognitiva las que se enfocan en lo conceptual (objetos o situaciones no presentes). El lenguaje concreto, propio de las preguntas de baja demanda cognitiva cumple la función de proveer al estudiantado con conocimiento conceptual básico, mientras que las preguntas de mayor demanda cognitiva permiten extender el conocimiento concreto a un nivel mayor de procesamiento (van Kleeck et al., 1997).

Theobald y Kultti (2012) aportan que más allá del tipo de pregunta, es necesario prestar más atención al momento en que se formulan y que las niñas y los niños puedan coproducir las interacciones en lugar de ser siempre quienes responden.

Hay toda otra serie de estudios que han desarrollado herramientas para clasificar el estilo interaccional docente según cierto tipo de comportamientos, que van más allá de las preguntas en sí. Algunos de estos instrumentos conocidos son el CLASS (La Paro, Pianta y Stuhlman, 2004) o el Teaching Styles Ratings Scale (Kruif, Ridley, McWilliam y Wakely, 2000), que permite identificar dos tipos principales de estilos docentes: los controladores 
directivos y los elaborativos. El estilo elaborativo implica la habilidad de expandir, a partir del compromiso infantil, sin elicitar comportamiento. La escala mide la frecuencia con la que aparecen los comportamientos ligados a esta cualidad elaborativa. Este estilo interaccional involucra una serie de estrategias lingüísticas por parte de las docentes que incluyen reformulaciones y elaboraciones (Roediger y Pyc, 2012), preguntas abiertas (Albanese y Antoniotti, 1997), afirmaciones y repeticiones de una emisión infantil para incorporarla al discurso compartido.

Otra serie de trabajos ha tipificado estilos de lectura de cuentos de progenitores a sus hijos o hijas: entre ellos, Haden, Reese y Fivush (1996) describieron tres estilos de lectura según el contenido de expresiones extra-textuales que empleaban las madres durante la lectura de cuentos. El primer estilo enfatizaba las descripciones, el segundo estilo, la comprensión, y el tercero, la participación infantil durante la lectura. En el ámbito del aula, Albanese y Antoniotti (1997) evaluaron el efecto del estilo interactivo docente en la comprensión infantil. Caracterizaron las intervenciones en dos tipos de estilos: el narrativo y el dialógico y llegaron a la conclusión de que el dialógico estaba asociado a mayor comprensión infantil. Mientras que el estilo narrativo fue caracterizado como aquel en el cual las docentes formulaban pocas preguntas, sobre todo de tipo retórico, el estilo dialógico se caracteriza por la combinación de una amplia diversidad de tipos de preguntas durante la lectura del cuento: de verificación, personales, de opinión, de elicitación de inferencias y preguntas enciclopédicas.

Más allá de cuáles son las estrategias o el estilo interactivo que más oportunidades de desarrollo parece ofrecer a a la niñez, un dato muy importante para esta investigación es que tanto en el ámbito de la escuela, como en el ámbito del hogar, numerosos trabajos han mostrado que estas instancias parecen ser escasas. Solamente un porcentaje mínimo del número total del habla adulta destinada a la niñez se puede clasificar como de abstracción de alto nivel. Predominan siempre las categorías descritas como de abstracción de bajo nivel y de pensamiento concreto (identificar cuestiones, clarificar, llamar la atención infantil), los intercambios son más bien monológicos enfocados en preguntas simples de baja demanda cognitiva (Chen y Sonja de Groot, 2014; Durden y Dangel, 2008) y las preguntas abiertas resultan poco frecuentes (Siraj-Blatchford y Manni, 2008; Pence et al., 2008). Massey et al. (2008), no obstante, encontraron que si bien las preguntas con mayor demanda cognitiva representaban solo el $10 \%$ del habla docente dirigida a estudiantes de sectores bajos y que 
predominaban las preguntas de gestión de la clase, este patrón se alteraba en las situaciones de lectura en las que predominaban las preguntas de mayor demanda cognitiva.

El único contraste con estos hallazgos lo presentan Pentimonti y Justice (2010) en su estudio sobre el tipo de intervención de 5 docentes. En él mostraron que se valían en el $96 \%$ de los casos de estrategias de andamiaje de bajo soporte, como generalizaciones, razonamientos y predicciones, en las que la persona infante resuelve casi sin ayuda las tareas que implicarían mayor demanda cognitiva. Sugieren que estudiantes con menor desarrollo lingüístico se beneficiarían más de estrategias de alto soporte, con menor demanda cognitiva.

\subsection{La formación de educadores orientada a favorecer el desarrollo infantil}

Muchos trabajos han resaltado la correlación entre el empleo de las estrategias optimizadoras del desarrollo que mencionábamos antes y la preparación de docentes y recomiendan la formación continua (Girolametto, Weitzman y Greenberg, 2003; Neuman, 2008; Wasik, Bond y Hidman, 2006). Fukkink y Lont (2007), por ejemplo, presentan una síntesis de 16 estudios publicados que llegan a estas conclusiones. Otros señalan que para poder brindar esta educación de calidad, las y los educadores de nivel inicial deben tener una alta capacitación en relación con el desarrollo infantil y el lenguaje, deben saber qué enseñar y cómo enseñarlo (Dickinson y Brady, 2006). En lectura de cuentos, puntualmente, los trabajos de Whitehurst y su equipo (Whitehurst et al., 1994) han venido demostrando desde hace años que un programa de lectura compartida produce cambios sustanciales en las habilidades lingüísticas infantiles de sectores pobres. Wasik et al. (2006), basándose en el programa de Whitehurst et al. (1994), entrenaron a docentes de escuelas públicas de barrios desfavorecidos en formulación de preguntas y otros modos de intervención y $70 \%$ de las maestras cambiaron el modo de intervención durante la lectura con beneficios para el desarrollo infantil.

La gran cantidad de trabajos de Neuman de los últimos 20 años (Neuman, 1996; Neuman, 2008; Neuman y Cunningham, 2009) viene mostrando que la capacitación y el trabajo conjunto con especialistas que modelan, construyen confianza (Herll y O'Drobinak, 2004), escuchan sin dictar la respuesta correcta (Guiney, 2001) y proporcionan feedback descriptivo apoyado en eventos observables (Gallacher, 1997) produce cambios en las prácticas docentes que conllevan mayores oportunidades para el desarrollo infantil. Neuman (2008), que en 1999 había analizado 500 casos en barrios pobres, más recientemente lo 
demostró a través de un estudio en el que comparó tres grupos de docentes con tres modalidades de formación: uno con un curso y acompañamiento en las prácticas, otro con curso sin acompañamiento y otro sin curso. El grupo que tuvo curso y acompañamiento en las prácticas fue el que más correlacionó con la calidad en la enseñanza en el postest.

Van Kleeck, Vander Woude y Hammett Price (2006), por su parte, compararon dos grupos de infantes, uno con intervención en la que los adultos formulaban preguntas literales e inferenciales y otro sin intervención. Mostraron el impacto de una intervención en lectura de 15 minutos dos veces por semana, a lo largo de 8 semanas, en las habilidades de estudiantes preescolares con dificultades en lenguaje. Dieron cuenta así, al igual que otros trabajos (Pentimonti y Justice, 2010), de la importancia de formular ambos tipos de preguntas. Las investigaciones en Argentina están en línea con todos estos hallazgos (Borzone y Rosemberg, 1994; Manrique y Borzone, 2012; Manrique y Sanchez Abchi, 2015; Manrique y Sanchez Troussel, 2017; Rosemberg y Silva, 2009).

Ahora bien, este tipo de formación no es simple. Como se ha planteado en otros trabajos, no solo la tarea de comprensión de un cuento ha demostrado ser cognitivamente demandante para cualquier infante (Kintsch, 2005; Manrique y Borzone, 2010), sino que la intervención docente para facilitar la comprensión infantil también lo es (Manrique, 2011). La tarea del adulto en este contexto puede ser definida como un andamiaje que permite el trabajo en la zona de desarrollo potencial (Vygotsky, 1978). En otras palabras, durante la lectura compartida de cuentos, el estudiantado tiene la oportunidad de actuar en un nivel más avanzado que en su actividad independiente, con el acompañamiento de la persona adulta que funciona como un mediatizador del texto (Manrique y Borzone, 2010; Manrique y Sanchez Troussel, 2017) y realiza con el estudiantado lo que este aún no puede solo.

Desde el punto de vista lingüístico y cognitivo, el hecho de que se refiere a personas, objetos y lugares ausentes implica un mayor nivel de abstracción comparado con las conversaciones durante otras actividades (Deleau, Gandon y Taburet, 1993). Además, poner en palabras los procesos que se llevan a cabo para comprender el cuento, le permite al adulto guiar estos procesos e influir en ellos (Manrique y Sanchez Troussel, 2017). La comprensión que la persona adulta tiene del cuento, el modo que tiene de transmitirlo en cuanto a prosodia y el modo en que interpreta las dificultades que el estudiantado va encontrando en el proceso, incide en el apoyo que pueda proporcionar a la conformación de la representación mental individual del texto (Manrique y Borzone, 2010). 
Asimismo, la lectura conjunta de cuentos implica un alto nivel de intersubjetividad entre los participantes. Requiere de la persona adulta que pueda ubicarse en el punto de vista del infante para poder comprender qué información le falta y, teniendo en cuenta el texto a leer, proporcionar las preguntas adecuadas que le permitan conectar la nueva información que el texto proporciona con lo que ya sabe (Manrique y Sanchez Troussel, 2017; Dickinson y Porche, 2011).

Ahora bien, el apoyo al desarrollo infantil durante la lectura de cuentos por parte de los adultos ha sido relacionado en estudios en diversos países, con el nivel educativo de estos. En el ámbito del hogar estos trabajos muestran que los progenitores con mayor educación ofrecen entornos más favorables para la alfabetización (Hammet et al., 2003). Wu y Honig (2010) mostraron este resultado en su estudio con madres taiwanesas, mientras que González, Rivera, Davis y Taylor (2010) lo encontraron en familias latinas y afroamericanas y Melissa-Halikiopoulou y Natsiopoulou (2008) en familias griegas.

Weigel, Martin y Bennett (2006) y DeBaryshe (1995), por su parte, encontraron que las rutinas alfabetizadoras estaban asociadas a las creencias de quienes están a cargo de la crianza y que las y los infantes pertenecientes a las familias que empleaban estas rutinas mostraban luego más interés en el mundo letrado, mientras que a las personas con menos formación, leer les resulta menos relevante (Torr, 2004). Complementando esta idea, Natsiopoulou, Souliotis y Kyridis (2006) encontraron que había una asociación entre el número de interacciones extra-textuales y las técnicas de narración de las personas adultas, tal que cuando la técnica es de narración oral en lugar de lectura del cuento, tienen lugar menos intercambios extra-textuales. Además, estos autores encontraron que el método del relato era escogido principalmente por padres y madres que tenían menos años de estudios. Deducen de este estudio que la forma de narrar el cuento depende de la formación académica de cada narrador, tal que a mayor formación, más probabilidades de leer el cuento, mientras que cuanto menor es la formación más se prefiere la narración oral del cuento sin lectura.

Resumiendo lo hasta aquí planteado, a pesar de que se conoce mucho acerca de cuáles serían las prácticas lingüísticas favorecedoras del desarrollo y lo necesarias que son, especialmente para la niñez de sectores socioculturalmente desfavorecidos, los trabajos que las estudian en contexto han mostrado que son escasas en las aulas infantiles (Chen y Sonja de Groot, 2014; Dickinson, Darrow y Tinubu, 2008; Durden y Dangel, 2008; Hammett Price et al., 2003; Massey et al., 2008; Pence et al., 2008; Siraj-Blatchford y Manni, 2008). 
No solo las prácticas lingüísticas favorecedoras del desarrollo son escasas en las aulas, también son escasos los trabajos que documentan el cambio en el empleo de estrategias que no son optimizadoras del desarrollo a otras que sí lo son. Este hecho pone en evidencia que el desarrollo de habilidades adultas que permiten emplear estas estrategias es arduo. Algunos resultados de investigaciones en esta línea son los de Souto-Manning (2010); Weitzman, Girolametto y Greenberg (2006); Wasik et al., (2006) y los nuestros en Argentina (Manrique y Borzone, 2012; Manrique y Sanchez Abchi, 2015; Manrique y Sanchez Troussel, 2017). En el estudio de Souto-Manning (2010), por ejemplo, se documenta cómo dos maestras del programa Head Start atravesaron un proceso transformativo de reconceptualización y de cambio en sus prácticas para incorporar estudiantes de familias africanas. En los trabajos realizados en Argentina, por su parte, se muestran algunas dificultades del proceso de transformación de los modelos mentales de docentes en ejercicio, y los cambios en las prácticas pedagógicas, relacionados con dispositivos de formación específicos que abordan estas dificultades.

Teniendo en cuenta todas estas ideas, antecedentes y espacios de vacancia, diseñamos un dispositivo de formación para favorecer el desarrollo de habilidades que les permiten a educadoras docentes y no docentes la construcción de un andamio al proceso de comprensión infantil, con el objetivo de estudiar precisamente el cambio en las intervenciones docentes.

\section{Procedimientos metodológicos}

\subsection{Enfoque}

El diseño de la investigación responde a un enfoque exploratorio de tipo mixto. Motivó esta decisión el objetivo del estudio que era comparar el impacto de la formación en las intervenciones en el aula de dos grupos de educadoras diferentes. Para realizar esta comparación era necesario sistematizar los datos extraídos en uno y otro grupo, complementando los procedimientos metodológicos de los métodos cualitativos y cuantitaivos, como se podrá apreciar más adelante en el apartado de procedimiento de análisis de la información (3.5). La combinación de procedimientos de ambos métodos nos permite valernos de la riqueza de unos y otros: en este caso, puntualmente, nos da la posibilidad de comparar los resultados obtenidos en ambos grupos sin perder la profundidad, la contextualización y la riqueza interpretativa. Asimismo, la obtención de datos por vías 
diversas y los diferentes procedimientos de análisis nos permiten la triangulación que da lugar a una interpretación lo más amplia posible del fenómeno en estudio.

\subsection{Participantes}

Participaron de este estudio 5 maestras (M) y 5 madres cuidadoras (MC) de salas de 3 a 5 años, pertenecientes a dos instituciones educativas de la provincia de Buenos Aires, Argentina, que atienden alrededor de 280 niños y niñas en edad preescolar en situación de pobreza. Ambas instituciones se encuentran subvencionadas por la Iglesia Católica, lo que implica para las familias la colaboración económica con una cuota módica mensual. Las participantes fueron informadas acerca del proyecto previamente y se comprometieron por voluntad propia a asistir a los encuentros de formación y entrevistas. Se les aseguró compromiso de confidencialidad. Todas llevaban más de 4 años en cada una de las instituciones y más de 10 años de experiencia en educación infantil.

Las 5 madres cuidadoras participantes fueron seleccionadas dentro del barrio de pertenencia de quienes participaron, por sus aptitudes para el cuidado infantil. Comparten con este grupo muchos de sus códigos culturales. Con respecto a su nivel educativo, 3 de ellas no han completado sus estudios secundarios y 2 lo han hecho recientemente. La función asistencial es la que ellas mismas consideran como la predominante en su tarea. Las 5 maestras, por su parte, fueron seleccionadas para su tarea dentro de la Iglesia debido su curriculum, en primer lugar, y por las recomendaciones que traen de otros trabajos. Pertenecen a niveles socioculturales y económicos más altos que el alumnado. Con respecto a su nivel educativo, además de su título docente, cuentan con una formación heterogénea y desigual que responde a sus intereses y oportunidades. Cada una declara haber realizado por lo menos un curso de perfeccionamiento por año, para sumar puntaje para la carrera docente y actualizarse.

\subsection{Procedimientos de recolección de información empírica}

El corpus se conformó a partir de la video-filmación y posterior transcripción en detalle de dos situaciones de lectura de cuentos al grupo de infantes que cada una de las participantes tenía a cargo, una previa y una posterior a la participación en el dispositivo de formación, conformando un total de 20 situaciones; 10 previas y 10 posteriores al dispositivo de formación. Las situaciones de lectura de cuentos fueron realizadas en los meses de agosto y octubre, con una cantidad similar de niñas y niños de 4 y 5 años presentes en todos 
los casos (entre 18 y 25). La selección de los cuentos estuvo a cargo de cada una de las participantes. El largo de la filmación dependió del largo de la actividad de lectura de cuentos, decidido por cada educadora, que fue filmada de modo completo. El análisis de los datos se realizó en el año 2018.

\subsection{Los dispositivos de formación}

Se entiende por dispositivo de formación un artificio complejo creado con un fin formativo, que se adapta a las características de los y las participantes. Los dos dispositivos diseñados y puestos en juego tenían como objetivo general promover el desarrollo de habilidades que dieran lugar al desarrollo de un andamiaje durante la lectura de cuentos que potenciara el desarrollo cognitivo infantil. Se buscó lograr este objetivo a través del análisis del propio desempeño generado en dos instancias: individual y grupal.

La instancia grupal del dispositivo de formación tomó la forma de un taller de análisis de casos, a través de dramatizaciones de situaciones de lectura y visualizaciones en video de maestras leyendo cuentos. Se complementó con instancias expositivas en las que se presentaron nuevos conceptos acerca de los procesos cognitivos infantiles para comprender un cuento y sus dificultades. Y se realizaron ejercicios que permitían ensayar posibles modos de intervención para facilitar dichos procesos.

Esta instancia grupal contó con 5 reuniones de 1, 30 horas, en el caso de las maestras y 9 reuniones de 1 hora para las madres cuidadoras, en el lapso de tres meses (de agosto a octubre). Esta diferencia se debió a las posibilidades de cada uno de los grupos de acuerdo con sus otras actividades cotidianas en la institución.

La instancia individual del dispositivo de formación contó con dos reuniones por cada participante con la formadora, de 1 hora de duración. Consistió en el análisis de una situación de lectura de cuentos propia, que había sido registrada en video por la coordinadora, transcripta y entregada previamente a cada una de las participantes. El análisis fue realizado por cada una de las participantes de manera individual, bajo la supervisión de la coordinadora, con el apoyo de los conceptos teóricos y pragmáticos desarrollados en los encuentros grupales antes descriptos y con una guía de preguntas proporcionada por la formadora. Esta guía incluía preguntas para analizar la situación de lectura en relación con dimensiones: 
- Instrumentales, como: ¿Qué criterio usaste para elegir el cuento? ¿Cómo lo introdujiste? ¿Cuántas veces te detuviste durante la lectura? ¿Qué función cumplió esa pausa? ¿Qué características tuvieron cada una de tus intervenciones y particularmente las preguntas que formulaste?

- Sociales, por ejemplo: ¿Cómo se distribuyeron los turnos de habla? ¿Cómo se caracteriza tu liderazgo? ¿De qué manera se mantuvo la disciplina en el aula? ¿Qué tipo de roles aparecieron en la situación analizada entre el estudiantado?

- Psíquicas, como por ejemplo: ¿Se observa predominio de lo emocional o de lo racional? ¿Qué emociones aparecieron en esta situación? ¿Qué podes decir del deseo de aprender y del deseo de enseñar en esta situación? ¿Qué se puede vislumbrar acerca de la tolerancia a la frustración propia de todo aprendizaje?

Luego de realizada la tarea de análisis cada participante se reunía con la formadora para compartir el análisis realizado.

\subsection{Procedimiento para el análisis de la información}

Con el fin de comparar las situaciones de lectura de cuentos previas y posteriores al dispositivo de formación, desde un enfoque exploratorio de tipo mixto, se consideraron como unidad de análisis las predicaciones de las maestras que no constituyeran parte del texto leído, también llamado habla extratextual (van Kleek et al., 1997). La predicación fue definida como la relación unívoca entre una frase nominal y un verbo finito.

En primer lugar, se cuantificaron las predicaciones y se realizó una prueba estadística de Chi-cuadrado para establecer la significatividad estadística de la relación entre las variables habla extratextual del grupo de educadoras (MC o M) - momento previo o posterior al dispositivo de formación para un nivel de confianza de 95\%. Se calculó, además, el grado de asociación entre variables mediante la prueba phi.

En segundo lugar, se codificaron las predicaciones recurriendo a un sistema de categorías previamente elaborado a partir del Método Comparativo Constante (Glaser y Strauss, 1967) y ya presentado en otros trabajos (Manrique y Borzone, 2012) que se inspira en el previamente desarrollado por van Kleek et al. (1997). En el sistema se consideran las predicaciones de las educadoras según el tipo de procesamiento cognitivo que involucran, tal como se muestra en la Tabla 1. La demanda de procesamiento cognitivo guarda relación con el nivel de abstracción al que los trabajos antecedentes aluden, y que ya hemos referido previamente (Whitehurst et al., 1994). Indica el tipo de esfuerzo o grado de dificultad que 
requiere cada pregunta o comentario para poder ser procesado cognitivamente. La hipótesis que subyace, basada en la teoría sociocultural (Vygostsky, 1978), es que un entorno que ofrece tareas con altas demandas cognitivas y oportunidades para resolverlas con el adecuado andamiaje, favorece el desarrollo infantil.

Tabla 1. Sistema de categorías para clasificar las predicaciones del habla extratextual de ambos grupos de educadoras (MC y M) según la demanda de procesamiento cognitivo que plantean

Nivel 1: Predicaciones que requieren de la identificación y la etiquetación de objetos o personajes. (¿Dónde está el osito ahora acá? (señala imagen) / ¿Cuál es el perrito?).

Nivel 2: Predicaciones que implican la descripción de objetos, personajes o eventos, o la focalización en algunos haciéndolos salientes. (¿Dónde se había subido el osito? (muestra la ilustración). / ¿Qué está haciendo acá el osito? (señala al osito en la jaula).

Nivel 3: Predicaciones que incluyen pistas para las respuestas o la misma respuesta se encuentra presente en la pregunta. Pueden ser respondidas al azar o por lectura de pistas prosódicas (preguntas cerradas o que involucran la selección de una alternativa de entre dos (¿Estaba triste o estaba contento?).

Nivel 4: Predicaciones que implican la recuperación de información proporcionada por el texto o el conocimiento del mundo de los niños (Miren, miren al pingüino ¿Qué le dijo el pingüino?/ ¿Y tienen alas las jirafas?)

Nivel 5: Predicaciones que involucran manipular u operar con información activada: establecer relaciones (asociaciones, comparaciones) y realizar inferencias. (¿Ven? hay que hacerle caso a la mama./ ¿Porqué pensás que quería ir a buscar una mamá?)

\section{Predicaciones no clasificables respecto de su demanda cognitiva:}

a- De gestión de la clase: Cumplen funciones no relacionadas con el procesamiento del texto, como mantener, preservar o gestionar la comunicación en el grupo, o la disciplina, evaluar, motivar, asignar turnos, llamar la atención, etc.

b- Incompletas (Incompletas, frases inaudibles o superpuestas, frases incomprensibles).

Fuente: Elaboración propia a partir de Manrique y Borzone, 2012.

En tercer lugar, se contabilizó la frecuencia de aparición de cada uno de los tipos de predicaciones según la demanda de procesamiento que involucraban, en las situaciones previas y posteriores en cada situación de lectura de cuentos registrada. Por último, se comparó la frecuencia de aparición de predicaciones con diferente nivel de demanda cognitiva entre las situaciones previa y posterior de las maestras y de las madres cuidadoras por separado y entre sí.

Para complementar este análisis se realizó una breve descripción de los cuentos leídos y un análisis descriptivo a partir de una categoría a la que denominamos dinámica de lectura. La definimos como la particular forma que toma la situación de lectura de cuentos considerando el uso del texto (lectura literal o modificada), del soporte libro y de sus ilustraciones (si se muestran las ilustraciones y cuándo) y los momentos en los que tiene lugar el habla extratextual. 


\section{Resultados}

\subsection{Modificaciones en el habla extratextual luego de un dispositivo de formación en madres cuidadoras (MC) y maestras (M)}

Enfocaremos, primero, en el cambio observado en los grupos de MC y M en relación con la extensión del habla extratextual (promedio de predicaciones que no constituyen la lectura literal del texto). Luego, atenderemos al cambio observado en la cantidad de predicaciones según el tipo de procesamiento cognitivo demandado a las personas infantes durante la lectura del cuento por parte de ambos tipos de educadoras antes y despues del dispositivo de formación.

Tabla 2. Promedio de predicaciones del habla extratextual previo y posterior al dispositivo de formación en los grupos de educadoras (MC y M) en Argentina

\begin{tabular}{|c|r|r|}
\hline \multirow{2}{*}{ GRUPO DE EDUCADORAS } & \multicolumn{2}{|c|}{ MOMENTO } \\
\cline { 2 - 3 } & \multicolumn{1}{|c|}{ PREVIO } & POSTERIOR \\
\hline Grupo de MADRES CUIDADORAS (MC) & 61,3 & 198,6 \\
\hline Grupo de MAESTRAS (M) & 287 & 278,2 \\
\hline
\end{tabular}

Fuente: Elaboración propia, 2018

Con el propósito de examinar si existía una relación entre el grupo de educadoras y la cantidad de predicaciones extratextuales antes y despues del dispositivo de formación, se aplicó la prueba chi-cuadrado. Los resultados indicaron una asociación significativa entre la cantidad de predicaciones antes y después del dispositivo y el grupo de educadoras. ( $X^{2}(1$, $\mathrm{N}=4125)=270,35, \mathrm{p}<.05$. Como puede observarse son las madres cuidadoreas las que más progresaron.

Asimismo, se encontró una relación estadísticamente significativa, moderadamente fuerte $(r \phi=-0.256, p<0.05)$, entre el la cantidad de predicaciones antes y despues del dispositivo de formación y el grupo de educadoras.

Tal como muestra la Tabla 2, la cantidad de habla extratextual, medida en promedio de predicaciones, en el grupo de MC se triplicó si se comparan las situaciones previa y posterior. En el grupo de $\mathrm{M}$, en cambio, se redujo levemente la extensión del habla extratextual. Si comparamos ambos grupos, si bien la diferencia entre ambos se redujo notablemente en la situación posterior a la formación, el grupo de $\mathrm{M}$ continúa con un número mucho mayor de predicaciones que el grupo de MC.

A continuación, en la Tabla 3, presentamos los resultados de la comparación entre las intervenciones durante la lectura de cuentos de las M y las MC (el habla extra.textual), antes y después de participar en el dispositivo de formación. 
Tabla 3. Porcentaje de predicaciones según el tipo de procesamiento cognitivo demandado a través del habla extra-textual por grupo de maestras antes y después del dispositivo de formación

\begin{tabular}{|c|c|c|c|c|}
\hline Momento & \multicolumn{2}{|c|}{ Previo al dispositivo } & \multicolumn{2}{|c|}{ Posterior al dispositivo } \\
\hline $\begin{array}{l}\text { Grupos de } \\
\text { educadoras }\end{array}$ & Maestras & $\begin{array}{l}\text { Madres } \\
\text { cuidadoras }\end{array}$ & Maestras & $\begin{array}{l}\text { Madres } \\
\text { cuidadoras }\end{array}$ \\
\hline Gestión & $40,5 \%$ & $33,4 \%$ & $34,5 \%$ & $24,4 \%$ \\
\hline Incompletas & $5,7 \%$ & $2,5 \%$ & $6,3 \%$ & $7,5 \%$ \\
\hline Nivel 1 & $3,5 \%$ & $25,5 \%$ & $4,8 \%$ & $8,5 \%$ \\
\hline Nivel 2 & $5,5 \%$ & $12 \%$ & $6,2 \%$ & $13,6 \%$ \\
\hline Nivel 3 & $11 \%$ & $4,5 \%$ & $7,3 \%$ & $6,5 \%$ \\
\hline Nivel 4 & $26,7 \%$ & $16 \%$ & $27 \%$ & $28,7 \%$ \\
\hline Nivel 5 & $7,1 \%$ & $6,1 \%$ & $13,9 \%$ & $10,8 \%$ \\
\hline Total & 100 & 100 & 100 & 100 \\
\hline
\end{tabular}

Fuente: Elaboración propia, 2018

Como se observa en la Tabla 3, si atendemos, en principio, a los porcentajes en las situaciones previas a la realización del dispositivo de formación, observamos que durante las situaciones de lectura de cuentos previas a la formación, predominan las predicaciones de gestión en ambos grupos de educadoras, pero en segundo lugar, las predicaciones de las $\mathrm{M}$ se ubican en el nivel 4, mientras que las de las $M C$ se ubican en el nivel 1. Las predicaciones ubicadas en el nivel 4 son las terceras más frecuentes para las MC. Es decir, que las predicaciones de las MC en general están ubicadas en los niveles más bajos. Esto estaría indicando un mayor nivel de abstracción en el habla dirigida a infantes de parte de las $M$ en la situación inicial, si las comparamos con las MC.

Cuando consideramos los valores de las situaciones posteriores a la formación, las predicaciones destinadas a la gestión de la clase siguen siendo las más frecuentes para las $M$, pero para las $M C$ las más frecuentes son las de nivel 4. Para las $M$, el nivel 4 se encuentra en segundo lugar, como en las previas a la formación, pero el nivel 5 ocupa el tercer lugar - en vez del nivel 3 como ocurría en la situación previa a la formación. Para las MC, despues de las predicaciones de nivel 4 se ubican, en frecuencia, las predicaciones de gestión de la clase, y luego las de nivel 2, y las de nivel 5. Las predicaciones de nivel 1 que eran tan frecuentes han disminuido a una tercera parte, luego de la formación.

Si comparamos los datos de las situaciones previas y las posteriores a la formación, en el caso de las M, observamos que el nivel 5 es el que más ha aumentado - casi al doble, seguido por el nivel 4 y el 2. El nivel 3 y las predicaciones de gestión de la clase se reducen. En el caso de las MC se observa que todos los porcentajes han sufrido aumento, menos el nivel 1. Resulta evidente que, mientras para las $\mathrm{M}$ la mayor diferencia entre las situaciones 
pre y post formación lo constituye el nivel 5, para las MC la diferencia grande está en el nivel 4 y 1 (que se reduce significativamente). Es decir, las diferencias entre pre y post son mucho mayores para las $\mathrm{MC}$, notables en los niveles 4, 1 y 5 , mientras que las $\mathrm{M}$ aumentan la frecuencia de todos los niveles levemente, excepto el nivel 5, en el cual se observa una diferencia importante, y el nivel 3 , en que se reduce la frecuencia.

Si comparamos el cambio en los grupos de M y MC, ambos son similares en cuanto a que las predicaciones destinadas a la gestión disminuyen, el nivel 2 aumenta y el nivel 5 aumenta notablemente, casi al doble. Las diferencias entre ambos grupos se concentran en el nivel 1, en que para las M se mantiene bajo en la situación pre y post formación, mientras que en las $\mathrm{MC}$, en que se observaba muy alto, se reduce a tres cuartas partes de su frecuencia en la situación post formación. Asimismo, el nivel 4 aumenta poco para las M y mucho para las MC.

Las diferencias entre ambos grupos se reducen en las situaciones posteriores, dando cuenta de un patrón similar de aumento concentrado en los niveles más altos de demanda cognitiva. La mayor diferencia que se observa en dicho patrón es que las predicaciones de gestión de clase implican un porcentaje mucho mayor del habla para las M y menor para las MC, cuya habla se concentra también en los niveles de baja demanda cognitiva (Nivel 1 y Nivel 2).

Es importante aclarar que estas diferencias refieren a las magnitudes de los porcentajes y que no se ha realizado una prueba estadística, por lo cual es necesario ser precavidos con su interpretación. Resumiendo, podemos señalar como lo más relevante que las intervenciones que involucran un mayor nivel de procesamiento cognitivo han aumentado después de la formación en ambos grupos de educadoras. Este dato puede ser interpretado como una oferta de mayores oportunidades de desarrollo infantil, luego de la formación. Podríamos pensar, pues, que la formación ha tenido un impacto en ambos grupos. Este resultado muestra la potencialidad del dispositivo de formación, más allá del nivel educativo y procedencia social de las educadoras en formación. En segundo lugar, observamos que las diferencias iniciales entre las educadoras de ambos grupos son grandes, pero las diferencias en la situación posterior al dispositivo de formación son menores.

Estos resultados, en primer lugar, constituyen una evidencia de la existencia de diferencias de oportunidades de desarrollo infantil de diferentes procedencias sociales en tanto la niñez con mayor necesidad de educación en la escuela está siendo acompañada por educadoras que cuentan con menos acompañamiento y formación que las educadoras 
docentes. En segundo lugar, se pone de manifiesto que, si bien el desempeño de ambos grupos de educadoras parece haberse trasformado luego de la formación, aumentando las intervenciones de mayor demanda cognitiva, las educadoras no docentes - MC-, han modificado su intervención en mayor medida que las educadoras docentes. Tomamos este dato como un indicador de un impacto mayor del dispositivo de formación en la población de educadoras no docentes.

Estos hallazgos indican la viabilidad de la formación para el grupo de educadoras no docentes, que, más allá de su formación inicial más elemental, parecen haberse beneficiado de su participación en el dispositivo de formación. Lo que hemos encontrado contradice nuestra hipótesis acerca de la supuesta dificultad que encontrarían para beneficiarse de la formación. A su vez, abre una nueva pregunta acerca de la dificultad que parecen haber encontrado las $\mathrm{M}$ que han modificado su desempeño menos que las MC.

Es importante también resaltar que, aun habiendo aumentado las intervenciones consideradas favorecedoras del desarrollo infantil, las intervenciones de este tipo de las educadoras no docentes, al final del dispositivo, permanecen por debajo de las de las educadoras docentes.

\subsection{Modificaciones en la dinámica de lectura y características de los cuentos luego del dispositivo de formación}

Más allá de las diferencias en cantidad de habla y en el tipo de procesamiento cognitivo demandado al grupo de infantes, el análisis descriptivo de las situaciones de lectura y de los cuentos leídos arroja un dato fundamental a la hora de comprender el cambio entre situaciones, tanto si comparamos MC con M como si comparamos las situaciones previas y posteriores de las MC.

En la descripción de las situaciones de lectura nos fijamos en la dinámica de lectura de cuentos, considerando el uso del texto, del soporte libro y sus ilustraciones y los momentos en los que acontecía el habla extra textual.

Tabla 4: Cambio en la dinámica de lectura de cuentos en M y MC luego de la formación

\begin{tabular}{|l|l|l|}
\hline \multirow{2}{*}{ GRUPO DE EDUCADORAS } & \multicolumn{2}{|c|}{ MOMENTO } \\
\cline { 2 - 3 } & PREVIO & POSTERIOR \\
\hline MAESTRAS (M) & Lectura dialógica & Lectura dialógica \\
\hline MADRES CUIDADORAS(MC) & Narración oral del texto & Lectura dialógica \\
\hline
\end{tabular}

Fuente: Elaboración propia, 2018. 
Tal como muestra la Tabla 4, encontramos que el total de las M leían el texto literalmente e introducían comentarios durante la lectura misma y luego de la lectura destinaban un tiempo a la conversación en torno al cuento. De las cinco maestras, cuatro mostraban las ilustraciones luego de concluir la lectura de cada una de las páginas, esto daba lugar a comentarios infantiles y muchas de las preguntas que las maestras formularon también se ubicaron allí. La quinta de ellas leía el texto mientras mostraba las ilustraciones, con el libro de cara al estudiantado y su cuello torcido. Al terminar de leer y antes de pasar la página dejaba un tiempo para que el estudiantado continuara mirando las ilustraciones. Podemos considerar esta dinámica de lectura con el nombre que le han dado Whitehurst et al. (1994) de "lectura dialógica".

Las MC, en cambio, recurrieron las cinco a la narración oral del cuento en las situaciones previas a la formación. Dos de ellas realizaron la narración oral describiendo en modo presente las ilustraciones del libro, mientras las mostraban. Las otras tres restantes realizaron una mezcla de lectura de algunas partes de los cuentos literalmente y parafraseo de otras partes. Los comentarios infantiles también tenían lugar mirando las ilustraciones. Después del dispositivo de formación la dinámica de lectura del cuento de las $5 \mathrm{MC}$ fue la lectura dialógica.

Con respecto a los cuentos leídos, en el caso de las $M$ se trataba de libros especialmente escritos para infantes que estaban ordenados por edad, según los criterios evolutivos de las personas escritoras. Se encontraban todos en buenas condiciones y su única dificultad era, en algunos casos, el tamaño demasiado reducido que tenían para poder ser compartidos en un grupo tan numeroso. A diferencia de esto, los cuentos leídos por las MC en las situaciones previas al dispositivo eran cuentos que habían sido donados a la institución y que tenían las siguientes características: idioma español, no castellano (en un caso), páginas faltantes (en un caso), versión demasiado abreviada de un cuento tradicional (en otro caso). Demasiadas ilustraciones y poco texto (en otro caso). Es decir que los textos en sí no estaban acordes a la edad evolutiva del estudiantado. Las MC los tomaban de la biblioteca del aula y ya los conocían de modo que los adaptaban ellas mismas. En las situaciones de lectura posteriores al dispositivo de formación las MC seleccionaron de entre todos los cuentos que tenían, aquellos que pudieran leer y que estuvieran en condiciones. 


\section{Discusión}

El objetivo de este trabajo era considerar el impacto de un dispositivo de formación en las intervenciones durante la lectura de cuentos, en dos grupos de educadoras: docentes y no docentes. Lo primero que sorprende de los resultados es la gran diferencia inicial entre los grupos. ¿Qué nos pueden indicar estas diferencias en relación con la formación de ambos grupos de educadoras?

Es posible que el número modesto de interacciones extra-textuales de las MC durante las situaciones previas a la formación esté relacionado en parte con la dinámica de lectura de cuentos. En efecto, a diferencia de las M que leían el texto literalmente, las MC lo narraban oralmente. Si seguimos esta línea, la diferencia mayor encontrada entre M y MC radicaría en el posicionamiento discursivo de unas y otras. En efecto, la demanda cognitiva que conlleva la producción oral de narrativa es mayor que la que implica la lectura para un lector experto. La formulación de preguntas $\mathrm{o}$ de comentarios que favorezcan el procesamiento del texto durante la lectura también requiere de recursos cognitivos para su realización. Tal como se ha expuesto previamente, las intervenciones durante la lectura de un cuento suponen una alta complejidad cognitiva (Deleau et al., 1993; Manrique, 2011) e intersubjetiva (Manrique y Sanchez Troussel, 2017; Dickinson y Porche, 2011; Justice et al., 2008).

Desde el punto de vista de la economía cognitiva, en el caso de la narración oral, contaremos con menor cantidad de recursos disponibles para formular preguntas que si leemos un texto. De allí derivamos la hipótesis, que está en línea con los hallazgos de Natsiopoulou, Souliotis y Kyridis (2006) previamente citados, de que la limitación en las MC en el habla extratextual estaría relacionada, en parte, con el método empleado - en este caso relato oral en lugar de lectura del texto. Estos autores encontraron, al igual que el presente trabajo, que el método del relato era escogido principalmente por adultos que tenían menos años de estudios. Lo observado en el desempeño de las MC antes de la formación, parece indicar que en Argentina ocurre al igual que en otros países donde el tema fue estudiado: los adultos con menor educación ofrecen entornos menos favorables para la alfabetización (Hammet Price, van Kleek y Huberty, 2003; Wu y Honig, 2010; González, Rivera, Davis y Taylor, 2010; Melissa-Halikiopoulou y Natsiopoulou, 2008.)

Otra interpretación de los resultados es posible si incorporamos la teoría polifónica de la enunciación (Ducrot, 2001) para pensar las situaciones de lectura de cuentos de personas adultas a infantes. La idea básica de esta teoría consiste en plantear que en toda 
enunciación se hacen oír diferentes "voces", puntos de vista o personajes que pueden dialogar entre sí. Si extrapolamos esta idea para pensar la lectura de cuentos infantiles, podemos entenderla como una situación compleja en la que participan tres tipos de posicionamientos: el del mundo infantil, que constituye la audiencia, el del lector y un tercer posicionamiento: el del texto. Cada uno de estos posicionamientos, a su vez, puede portar diversas voces. Ahora bien, en la metodología de lectura del texto y habla extratextual, la voz del narrador del cuento se desdobla de la de la docente lectora del cuento. Es posible, por ejemplo, leer un párrafo y formular una pregunta que cuestione lo leído. La existencia de este tercer punto de posición -texto - con el cual los otros dos - persona lectora y audiencia infantil - se relacionan, permite un juego de toma de distancia con respecto al texto y habilita un mayor nivel de abstracción de los intercambios. Esta posibilidad polifónica no está presente en las situaciones en las que el cuento se comparte a través de una dinámica de lectura de narración oral, en la que el texto y los comentarios de quien lo lee aparecen bajo la misma voz.

Ahora bien, luego de la formación las MC, más allá de su nivel educativo bajo, fueron capaces de cambiar su dinámica de lectura por la lectura dialógica (Whitehurst et al., 1994), cambio que estuvo acompañado, como ya se mostró, de intervenciones de mayor demanda cognitiva. En términos de la teoría polifónica (Ducrot, 2001), las MC habrían incorporado, junto con este cambio de método de la narración oral a la lectura comentada con habla extra textual, la posibilidad que la polifonía les ofrece. Esto nos hace pensar que la diferencia casi inexistente entre $\mathrm{M}$ y $\mathrm{MC}$ registrada en las situaciones posteriores al dispositivo se relaciona en gran medida con este cambio de dinámica de lectura. Esto explica también por qué el impacto del dispositivo parece haber sido mayor para las MC, quienes parecen haberse beneficiado más de la formación que las $M$, al incorporar este nuevo método. A partir de la formación las MC fueron capaces, al igual que las maestras, al leer un cuento, de constituirse en un tercero que pregunta, cuestiona y comenta ese texto. Se trata de un proceso de transformación que va más allá de una serie de estrategias y alude a un nuevo modo de leer un cuento, a un posicionamiento discursivo nuevo que habilita procesos de abstracción, que son considerados favorecedores del desarrollo infantil (Dickinson y Smith, 1994; Curenton y Justice, 2004; Justice, Meier y Walpole, 2005; Rosemberg y Silva, 2009; van Kleeck et al., 2006; Wasik et al., 2006).

Esta lectura desde la teoría polifónica constituye, también desde el punto de vista teórico, una nueva explicación acerca de por qué la lectura de cuentos compartida parece 
ser tan útil para el desarrollo lingüístico y cognitivo infantil, en comparación con otras actividades que se realizan en el jardín de infantes (Massey et al., 2008; Borzone, 2005; Justice, Weber, Ezell y Bakeman, 2002; Piacente y Tittarelli, 2009; Hammett Price, van Kleeck y Huberty, 2009; Raikes et al., 2006).

Desde el punto de vista de la formación se abre otra discusión: ¿Cómo podemos entender que las maestras, de quienes esperábamos encontrar mayor disponibilidad a la reflexión sobre su práctica, y, por ende, mayor capacidad de transformación hayan cambiado menos que las MC? Es evidente que con los resultados del análisis realizado no podemos saber qué aspectos se habrán jugado para que las $\mathrm{M}$ no hayan modificado tanto como las MC su intervención. Lo único que podemos hipotetizar es que las MC, más allá de no haber tenido la oportunidad de revisar los fundamentos de sus intervenciones durante su formación previa, no parecerían haber tenido dificultades en realizar esa reflexión que el dispositivo de formación les ofreció, en este momento de su carrera. No habrían modificado sus intervenciones de no haber sido capaces de hacerlo. En efecto, el hecho de que hayan optado por una dinámica de lectura dialógica en lugar de su antigua dinámica de narración oral, da cuenta de que habrían modificado sus creencias en torno a la lectura de cuentos, su función en el desarrollo infantil y su modo de llevarla a cabo. De ser así, estaríamos reafirmando con Weigel, Martin y Bennett (2006) y con DeBaryshe (1995) el vínculo entre las creencias y las rutinas alfabetizadoras.

Más allá de esta hipótesis tentativa, la respuesta a esta pregunta acerca de la formación excede las posibilidades de este trabajo. Este límite debe ser pensado como un punto de partida para otros trabajos. Parece importante en futuras investigaciones considerar mayor información que nos permita llevar a cabo inferencias y comenzar a responder a la última pregunta que formulamos con mayor rigurosidad.

El trabajo tiene, además, otra serie de limitaciones que deben ser tenidas en cuenta. En primer lugar no estamos considerando los cuentos elegidos en detalle, aun sabiendo que la interacción lingüística entre personas adultas y niñez también parece afectarse por el tipo de texto (Borzone y Manrique, 2010; Neuman, 1996; Hammett Price et al, 2009). En relación con el andamiaje docente, como no estamos incluyendo el habla infantil en este particular estudio, no podemos saber si efectivamente la modificación en el tipo de intervenciones discursivas de las maestras y madres cuidadoras se estaba ajustando al nivel requerido por la niñez. Es por eso que tuvimos cuidado en no realizar inferencias en cuanto a la oportunidad de desarrollo que las situaciones de lectura ofrecen. En futuras investigaciones, 
será importante también prestar atención al momento en que se formulan las preguntas, tal como sugieren Reese y Cox (1999) y Theobald y Kultti (2012), así como a la respuesta infantil luego de formulada la pregunta, para observar la contingencia de la intervención adulta en relación con la persona pequeña, la adaptación a sus intereses y su línea de pensamiento, aspecto que se ha revelado como relevante (Bateman, 2013). En efecto, este autor en su estudio muestra que no basta con realizar preguntas abiertas, sino que lo importante es la escucha de la población infantil para poder valorar su contribución.

Tampoco resulta posible saber la relación entre las intervenciones de mayor y menor demanda cognitiva, solamente a partir del conteo de frecuencias de aparición de cada una de ellas. De modo que no podemos ver en qué medida estos tipos de preguntas se están relacionando o cómo se vinculan con las intervenciones infantiles. Consideramos que puede ser interesante estudiarlo en un futuro trabajo, siguiendo a autores como Pentimonti y Justice (2010), para quienes es importante pasar de preguntas de menor a otras de mayor demanda cognitiva, o combinar ambas para el beneficio infantil, sobre todo en sectores bajos.

El foco ha estado puesto, en este caso, en el cambio en el modo de intervenir que el dispositivo de formación parece haber impulsado, por una parte; y en la diferencia de este cambio entre ambos grupos de educadoras. Se trata de un trabajo centrado en la posibilidad de cambiar que tienen dos poblaciones de educadoras muy diferentes. Más allá de las limitaciones del estudio, nos parece importante subrayar la relevancia de los hallazgos de este estudio tanto para el ámbito de la formación docente, como para el del desarrollo lingüístico infantil.

\section{Conclusiones}

En este trabajo se muestra cómo en Buenos Aires, Argentina, las educadoras participantes con mayor formación - las M- muestran inicialmente un desempeño diferente en sus prácticas de lectura de cuentos, de aquellas educadoras que no han tenido formación -las MC-. Sin embargo, también se muestra cómo, después del dispositivo de formación, a pesar de no contar con experiencias formativas formales previas, y a pesar de que no cuentan con el hábito de la lectura, las MC fueron capaces de capitalizar la experiencia de formación de modo que se operan en sus prácticas transformaciones importantes, hasta tal punto que el desempeño en las situaciones de lectura que se observa por parte de las MC y de las M después del dispositivo de formación son similares. 
El hecho de que luego de la formación todas las educadoras docentes y no docentes hayan manifestado cambios en su desempeño en relación con el habla dirigida a la niñez durante la lectura, da cuenta de dos cuestiones. En primer lugar de que la formación produce efectos en el desempeño docente, como tantos otros trabajos lo vienen mostrando (Girolameto et al, 2003; Neuman, 1996, 2008; Neuman y Cunningham, 2009; Wasik et al, 2006; Fukkink y Lont, 2007; Whitehurst et al., 1994). En segundo lugar, da cuenta de la efectividad del dispositivo de formación $\mathrm{y}$, por ende, del empleo de videos y de la autoobservación que el dispositivo ofreció como modo de estimular la reflexión sobre la acción y el reposicionamiento docente. Estos resultados coinciden con lo identificado por un número importante de investigaciones que defienden este método de formación docente por sobre otros posibles (Barth-Cohen et al., 2018; Gelfuso y Denis, 2017; Assen et al., 2016).

Los resultados que muestran mayores cambios en las $M C$ que en las $M$ parecen estar indicando la potencial capacidad transformadora del dispositivo, como antes señalamos, así como sus propios límites. Es decir, que indica las condiciones de posibilidad de todos los sujetos para formarse, aspecto importante a la hora de plantearse si las MC se beneficiarían de un dispositivo, más allá de su formación inicial menor que la de las M. También nos pone sobre aviso con respecto a la existencia de diferencias que fundamentan la necesidad de pensar la formación de acuerdo con las condiciones particulares de los sujetos en formación.

Plantea también una serie de preguntas que quedan abiertas, en relación con la capacidad de cambiar, mayor para las MC que para las M. Consideramos que es necesario una profundización de este tema en futuros trabajos, por la relevancia que tiene para comprender los mecanismos de transformación en docentes que llevan muchos años en ejercicio.

Con respecto a la lectura de cuentos como actividad promotora del desarrollo infantil, por último, la incorporación de la idea de posicionamiento discursivo al leer un texto, que tomamos de la teoría polifónica (Ducrot, 2001) y la posibilidad de devenir tercero que la lectura dialógica permite, da lugar a continuar construyendo conocimiento acerca de los motivos por los cuales la lectura interactiva de cuentos, y no tanto su narración oral, resulta una de las vías posibles para optimizar las oportunidades de aprendizaje infantil. Permite, también, poner en visibilidad la importancia de la formación de personas que tienen esta tarea a cargo, como un modo de achicar la brecha entre niñas y niños de diferentes sectores socioculturales. 


\section{Referencias}

Albanese, Ottavia. y Antoniotti, Carla. (1997). Teacher Dialogue Style and Children's Story Comprehension. European Journal of Psychology of Education, 12(3), 249-59.

Assen, Hanneke, Meijers, Frans, Otting, Hans. y Poell, Rob. (2016). Explaining discrepancies between teacher beliefs and teacher interventions in a problem-based learning environment: A mixed methods study. Teaching and teacher education, 60, 12-23.

Barth-Cohen, Lauren, Little, Angela. y Abrahamson, Dor. (2018): Building Reflective Practices in a Pre-service Math and Science Teacher Education Course That Focuses on Qualitative Video Analysis. Journal of Science Teacher Education, 29(2), 83-101.

Bateman, Amanda. (2013). Responding to Children's Answers: Questions Embedded in the Social Context of Early Childhood Education. Early Years, 33(3), 275-288.

Blanchard- Laville, Claudine. (2013). Para una clínica grupal del trabajo docente. Revista del IICE, 34, 7-28.

Borzone, Ana María. y Rosemberg, Celia. (1994). El intercambio verbal en el aula: las intervenciones de los niños en relación con el estilo de interacción del maestro. Infancia y Aprendizaje, 17(67-68), 115-132.

Borzone, Ana María. (2005). La lectura de cuentos en el jardín infantil: un medio para el desarrollo de estrategias cognitivas y lingüísticas. Psykhe, 14(1), 192-209.

Borzone, Ana María. y Manrique, María Soledad (2010). El contexto cognitivo en situaciones de lectura de cuentos en el Jardín de Infantes. Revista Lenguaje, 38(1), 65-92.

Brouwer, Niels. y Korthagen, Fred. (2005). Can teacher education make a difference?. American Educational Research Journal, 42, 153-224.

Chen, Jennifer. y Sonja de Groot, Kim. (2014). The quality of teachers' interactive conversations with preschool children from low-income families during small-group and large-group activities. Early Years: An International Research Journal, 34(3), 271-288.

Curenton, Stephanie. y Justice, Laura. (2004). African American and Caucasian preschoolers' use of decontextualized language: Literate language features in oral narratives. Language, Speech, and Hearing Services in Schools, 35, 240-253.

Darling-Hammond, Linda. (2006). Preparing teachers for a changing world: What teachers should learn and be able to do. San Francisco, California, Estados Unidos: JosseyBass.

DeBaryshe, Bárbara. (1995). Maternal belief systems: Linchpin in the home reading process. Journal of Applied Developmental Psychology, 16, 1-20.

Deleau, Michael, Gandon, Eva. y Taburet, Veronique. (1993). Semiotic mediation in guiding interactions with young children: the role of context and communication handicap on distanciation in adult discourse. European Journal of Psychology of Education, 8, 473486. 
Devescovi, Antonella. y Baumgartner, Ema. (1993). Joint-reading a picture book: Verbal interaction and narra-tive skills. Cognition and Instruction, 11, 299-323.

Dickinson, David. y Brady, Joanne. (2006). Toward effective support for language and literacy through professional development. En M. Zaslow y I. Martinez-Beck (Eds.), Critical issues in early childhood professional development (pp. 141-170). Baltimore, Estados Unidos: Brookes.

Dickinson, David, Darrow, Catherine. y Tinubu, Titilayo, Alí. (2008). Patterns of TeacherChild Conversations in Head Start Classrooms: Implications for an Empirically Grounded Approach to Professional Development. Early Education y Development, 19(3), 396-429.

Dickinson, David. y Porche, Michelle. (2011). Relation between Language Experi-ences in Preschool Classrooms and Children's Kindergarten and Fourth-grade Language and Reading Abilities. Child Development, 82(3), 870-886.

Dickinson, David. y Smith, Miriam. (1994). Long-term effects of preschool teachers'book reading on low-income children's vocabulary and story comprehension. Reading Reseach Quarterly, 29(2), 104-123.

Ducrot, Oswald. (2001). El decir y lo dicho. Buenos Aires, Argentina: Edicial.

Durden, Tonia Renee. y Dangel, Julie Rainer. (2008). Teacher-involved Conversations with Young Children during Small Group Activity. Early Years, 28(3), 251-266.

Fukkink, Ruben. y Lont, Anna. (2007). Does training matter? A meta-analysis and review of caregiver training studies. Early Childhood Research Quarterly, 22, 294-311.

Gallacher, Kathleen. (1997). Supervision, mentoring, and coaching. En W. P. J. McCollum y C. Catlett (Eds.), Reforming personnel in early intervention (pp. 191-214). Baltimore, Estados Unidos: Brookes.

Gelfuso, Andrea. y Dennis, Danielle. (2017) Video as Text of Teaching: Toward More Deliberate Literacy Field Experience Supervision. The Teacher Educator, 52(1), 57-74.

Girolametto, Luigi, Weitzman, Elaine. y Greenberg, Janice. (2003). Training day care staff to facilitate childrens' language. American Journal of Speech-Language Pathology, 12, 299-311.

Glaser, Barney y Strauss, Anselm. (1967). The Discovery of Grounded Theory: strategies for qualitative research. Nueva York, Estados Unidos: Aldine de Gruyter.

Gonzalez, Jorge, Rivera, Vanesa, Davis, Matthew. y Taylor, Aaron. (2010). Foundations of children's vocabulary development: The role of the home literacy environment. Early Childhood Services, 10, 55-72.

Guiney, Ellen. (2001). Coaching isn't just for athletes: The role of teacher leaders. Phi Delta Kappan, 82, 740-743. 
Haden, Catherine, Reese, Elaine. y Fivush, Robyn. (1996). Mothers'extratextual comments during storybook reading: stylistic differences over time and across texts. Discourse Processes, 21, 135-169.

Hammett Price, Lisa, Van Kleeck, Anne. y Huberty, Carl. (2003). Patterns of Parents' Extratextual Interactions during Book Sharing with Preschool Children: A Cluster Analysis Study. Reading Research Quarterly, 38(4), 442-468.

Hammet Price, Lisa, van Kleeck, Anne. y Huberty, Carl. (2009). Talk during book sharing between parents and preschool children: A comparison between storybook and expository book conditions. Reading Research Quarterly, 44, 171-194.

Hattie, John. (2009). Visible learning: A synthesis of over 800 meta-analyses relating to achievement. Londres, Inglaterra: Routledge.

Herll, Susan. y O'Drobinak, Brooke. (2004). Role of a coach: Dream keeper, supporter, friend. Journal of Staff Development, 25(2), 42-46.

Hermans, Hubert. y Hermans-Konopka, Agnieszka. (2010). Dialogical self theory: Positioning and counter-positioning in a globalizing society. Cambridge, Inglaterra: Cambridge University Press.

Justice, Laura, Mashburn, Andrew, Hamre, Bridget. y Pianta, Robert. (2008). Quality of language and literacy instruction in preschool classrooms serving at-risk pupils. Early Childhood Research Quarterly, 23(1), 51-68.

Justice, Laura, Weber, Sarah, Ezell, Hellen. y Bakeman, Roger. (2002). A sequential analysis of children's re-sponsiveness to parental print references during shared book-reading interactions. American Journal of Speech-Language Pathology, 11, 30-40.

Justice, Laura, Meier, Joanne. y Walpole, Sharon. (2005). Learning new words from storybooks: An efficacy study with at-risk kindergartners. Language, Speech, and Hearing Services in Schools, 36, 17-32.

Kintsch, Eileen. (2005). Comprehension theory as a guide for the design of thoughtful questions. Topics in Language Disorders, 25, 51-64.

Kruif, Renee, Ridley, Stephanie, Maher, Robin McWilliam. y Wakely, Melissa. (2000). Classification of teachers' interaction behaviors in early childhood classrooms. Early Childhood Research Quarterly, 15(2), 247-268.

La Paro, Karen, Pianta, Robert. y Stuhlman, Megan. (2004). The classroom assessment scoring system: findings from the prekindergarten year. Elementary School Journal, 104(5), 409-426.

Lonigan, Christopher. y Whitehurst, Grover. (1998). Relative efficacy of parent and teacher involvement in a shared-reading intervention for preschool children from low-income backgrounds. Early Childhood Research Quarterly, 13, 263-290.

Manrique, María Soledad. (2011). Las demandas lingüísticas y cognitivas de la lectura de cuentos en el Jardín de Infantes. Revista IRICE de Educación y Psicología, 22, 45-59. 
Manrique, María Soledad. y Borzone, Ana María. (2010). La comprensión de cuentos como resolución de problemas en niños de 5 años de sectores urbano - marginados. Revista Interdisciplinaria, 27(2), 209-228.

Manrique, María Soledad. y Borzone, Ana María. (2012). La formación transforma. El caso de una madre cuidadora. Revista Educere, 16(53), 171-186.

Manrique, María Soledad. y Sanchez Abchi, Verónica. (2015) Teacher's practices and mental models. Transformations through reflection on action. Australian Journal of Teacher Education, 40(6), 13-32.

Manrique, María Soledad. y Sanchez Troussel, Lorena. (2017). Andamiar la comprensión infantil durante la lectura de cuentos: Dispositivo de formación basado en el análisis del propio desempeño. En Silvia Romero y Sol Concha (Eds), Formación docente en el área de lenguaje. Experiencias en América Latina (pp. 295-331). Ciudad de México, México: 12 Editorial.

Mashburn, Andrew, Justice, Laura, Downer, Jason. y Pianta, Robert. (2009). Peer effects on children's language achievement during pre-kindergarten. Child Development, 80, 686702.

Massey, Susan, Pence, Khara, Justice, Laura. y Bowles, Ryan, Patrick. (2008). “Educators' Use of Cognitively Challenging Questions in Economically Disadvantaged Preschool Classroom Contexts. Early Education and Development, 19(2), 340-360.

Melissa- Halikiopoulou, Chrysoula. y Natsiopoulou, Triantafillia. (2008). Mothers Reading childrens'books to preeschoolers. A Greek Study. International Journal of Caring Sciences, 1(2), 74-85.

Mevorach, Miriam. y Strauss, Sidney. (2012). Teacher Educators' In-Action Mental Models in Different Teaching Situations. Teachers and Teaching: theory and practice, 18(1), 2541.

Natsiopoulou, Triantafillia, Souliotis, Mimis. y Kyridis, Argyris. (2006). Narrating and reading folktales and picture books: Storytelling techniques and approaches with preschool children. Early Childhood Research Practice, 8(1), 69-79.

Neuman, Susan. (1996). Children engaging in storybook reading: The influence of access to print resources, opportunity and parental interaction. Early Childhood Research Quarterly, 11, 495-513.

Neuman, Susan. (2008). Changing the odds for children at risk: Seven essential prin-ciples of education programs that break the cycle of poverty. Westport, CT, Estados Unidos: Praeger.

Neuman, Susan. y Cunningham, Linda. (2009). The Impact of Professional Development and Coaching on Early Language and Literacy Instructional Practices. American Educational Research Journal, 46(2), 532-566. 
Pence, Khara, Justice, Laura. y Wiggins, Alice. (2008). Preschool teachers' fidelity in implementing a comprehensive language-rich curriculum. Language, Speech, and Hearing Services in Schools, 39(3), 329.

Pentimonti, Jill. y Justice, Laura (2010). Teachers' Use of Scaffolding Strategies duringRead Alouds in the Preschool Classroom." Early Childhood Education Journal, 37, 241-248.

Perrenoud, Philippe. (2004). Desarrollar la práctica reflexiva en el oficio de enseñar: profesionalización y razón pedagógica. Barcelona, España: Graó.

Piacente, Telma. y Tittarelli, Ana María. (2009). La lectura de cuentos dialógica y las características de la interacción según estrato social de procedencia. Evaluar, 9, 1-18.

Pozo, Ignacio, Scheuer, Nora, Pérez Echeverría, María del Puy; Mateos, María del Mar; Martín, Elena. y De la Cruz, Monserrat. (2006). Nuevas formas de pensar la enseñanza y el aprendizaje. Las concepciones de profesores y alumnos. Colección Crítica y Fundamentos. Barcelona, España: Gráo.

Raikes, Hellen, Pan, Barbara, Alexander, Luze, Gayle, Tamis-LeMonda, Catherine, GrooksGunn, Jeanne. y Constantine, Jill. (2006). Mother-child bookreading in low-income families: Correlates and outcomes during the first three years of life. Child Development, 77, 924-953.

Reese, Elaine. y Cox, Adelle. (1999). Quality of adult book reading affects children's emergent literacy. Developmental Psychology, 35, 20-28.

Roediger, Henry. y Pyc, Mary. (2012). Inexpensive techniques to improve education: applying cognitive psychology to enhance educational practice. Journal of Applied Research in Memory and Cognition, 1(4), 242-248.

Rosemberg, Celia, Renata. y Silva, María Luisa. (2009). Teacher- children interaction and concept development in kindergarten. Discourse processes, 46, 572-591.

Siraj-Blatchford, Iram. (2010). Learning in the home and at school: How working class children 'succeed against the odds'. British Educational Research Journal, 36(3), 46382.

Siraj-Blatchford, Iram. y Manni, Laura. (2008). Would you like to tidy up now? An analysis of adult questioning in the English Foundation Stage. Early Years, 28(1), 5-22.

Souto, Marta. (2016). Los pliegues de la formación. Sentidos y herramientas para la formación docente. Buenos Aires, Argentina: Homo Sapiens.

Souto-Manning, Mariana. (2010). Challenging Ethnocentric Literacy Practices: $(\mathrm{Re})$ Positioning Home Literacies in a Head Start Classroom. Research in the Teaching of English, 45(2), 150-178.

Theobald, Maryanne. y Kultti, Anne. (2012). Investigating Child Participation in the Everyday Talk of Teacher and Children in a Preparatory Year. Contemporary Issues in Early Childhood, 13(3), 210-225. 
Torr, Jane. (2004). Talking about picture books: The influence of maternal education on fouryear-old children's talk with mothers and pre-school teacher. Journal of Early Childhood Literacy, 4, 181-210,

van Kleek, Anne; Gillam, Ronald; Hamilton, Lori. y McGrath, Cassandra. (1997). The relationship between middle-class parents' book - sharing discussion and their preschoolers' abstract language development. Journal of Speech, Language and Hearing Research, 40(6), 1261-1271.

Van Kleeck, Anne, Vander Woude, Judith. y Hammett Price, Lisa. (2006), Fostering literal and inferential language skills in Head Start preschoolers with language impairment using scripted book-sharing discussions. American Journal of Speech-Language Pathology, 15(1), 85-95.

Vygotsky, Lev Semionovich. (1978). Mind in society. The development of higher psychological processes. Cambridge, Mass, Estados Unidos: Harvard University Press.

Wasik, Barbara, Bond, Mary Alice. y Hindman, Annemarie. (2006). The Effects of a Language and Literacy Intervention on Head Start Children and Teachers. Journal of Educational Psychology, 98(1), 63-74.

Weigel, Daniel, Martin, Sally. y Bennett, Kymberley. (2006). Mothers' literacy beliefs: Connections with the home literacy environments and pre-school children's literacy development. Journal of Early Childhood Literacy, 6, 191-211.

Weitzman, Elaine, Girolametto, Luigi. y Greenberg, Janice. (2006). Adult Responsiveness as a Critical Intervention Mechanism for Emergent Literacy: Strategies for Early Childcare Educators. En L. Justice (Ed.), Clinical Approaches to Emergent Literacy Intervention (pp 127-178). San Diego, CA, Estados Unidos: Plural Publishing.

Whitehurst, Grover, Arnold, D., Epstein, J., Angell, A., Smith, M. y Fischel, J. (1994). A picture-book reading intervention in day care and home for children from low-income families. Developmental Psychology, 30, 679-689.

Whitehurst, Grover. y Lonigan, Christopher. (1998). Child development and emergent literacy. Child Development, 69, 848-872.

Winnicot, Donald. (1986). Realidad y juego. Buenos Aires, Argentina: Gedisa.

Wu, Chu Chu. y Honig, Alice Sterling. (2010). Taiwanese mothers' beliefs about reading aloud with preschoolers: Findings from the Parent Reading Belief Inventory. Early Child Development and Care, 180, 647-669. 
Revista indizada en

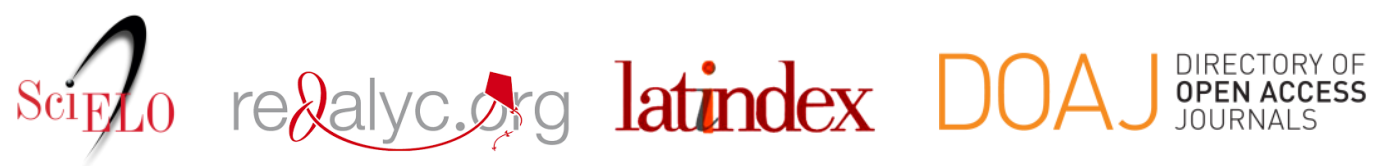

Distribuida en las bases de datos:

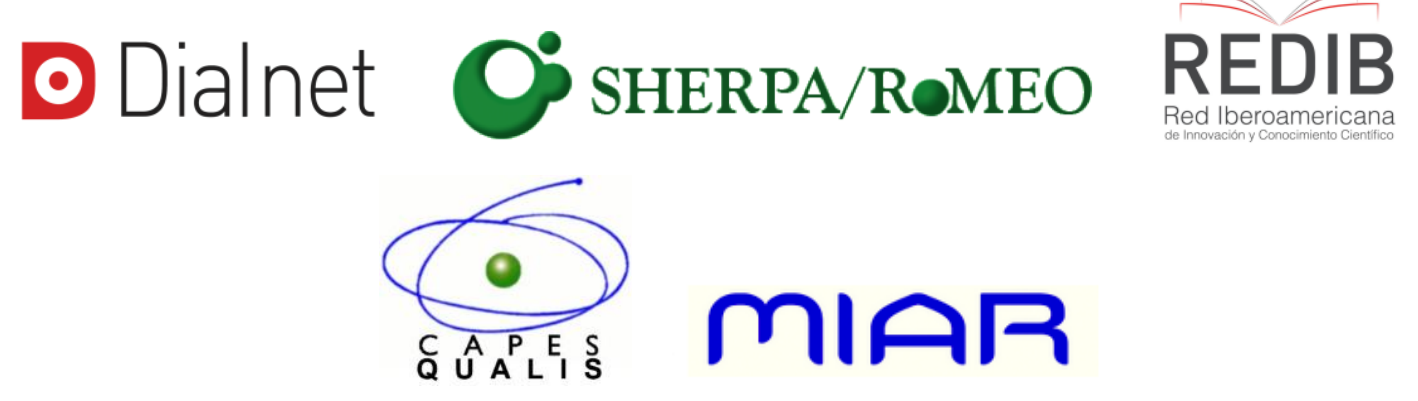

